\title{
ELECTRON STRUCTURE OF $d$-TRANSITION METALS AND THEIR ALLOYS ON THE BASIS OF EELS STUDY
}

\author{
H.B. KoLODZIEJ
}

Institute of Physics, Pedagogical University

Al. Armii Krajowej 13/15, 42-201 Częstochowa, Poland

Analysis of characteristic electron energy losses in reflection from the surface provides information on electron and band structure of the solid state. Study of transition metals, their alloys $(\mathrm{Fe}-\mathrm{N}, \mathrm{Zn}-\mathrm{Ni})$ and amorphous alloys $\mathrm{Fe}_{78} \mathrm{Si}_{9} \mathrm{~B}_{13}$ show that there are two subsets of electron gas characterized by different oscillation frequency. The presence of independent components of volume plasmons corresponding to $s$ and $d$ electrons is an evidence that despite collectivization both the subsets preserve individual features characteristic of the structure of an isolated atom. From the energy losses the following quantities are determined: the effective mass $(\mu)$ for particular electron subsets, the Fermi energy $\left(E_{\mathrm{F}}\right)$, the band widths $(\delta E)$ for $s$ and $d$ electrons and contribution of the subsets to the metal conductivity $(\gamma)$. The obtained results for metals are compared to analogous data for alloys both in crystalline and amorphous phase.

PACS numbers: 61.43.Dq, 71.45.Gm, 78.70.Bj, 79.20.Kz, 72.30.+q

\section{Introduction}

According to the Pines theory [1] the energy lost by an incident electron can be expressed by the relations

$$
\begin{aligned}
& \hbar \omega_{\mathrm{p}}=\hbar\left(\frac{4 \pi n_{0} e^{2}}{m_{0}}\right)^{1 / 2} \\
& \hbar \omega_{\mathrm{p}}=B\left(\frac{n \rho}{A}\right)^{1 / 2}
\end{aligned}
$$

where $n_{0}$ is the electron gas concentration, $e, m$ are the electron charge and mass, respectively, $B=\hbar e\left(4 \pi N_{\mathrm{A}} / m_{0}\right)^{1 / 2}=28.78 \mathrm{eV} \mathrm{cm}^{3 / 2}, N_{\mathrm{A}}$ is the Avogadro number, $\rho$ and $A$ are the density and atomic mass of a sample, $n$ is the electron number per atom. 
Experimentally determined electron energy loss may be expressed in terms of the electron effective mass $\mu$ as

$$
\Delta E=\hbar \omega_{\mathrm{p}}\left(m_{0} / \mu\right)^{1 / 2} .
$$

From Eqs. (2) and (3) we obtain

$$
\frac{\mu}{m_{0}}=\frac{B^{2} \rho n}{A(\Delta E)^{2}} \text {. }
$$

This relation allows to evalua' 'he effective mass $\mu$ if the energy loss $\Delta E$ is known.

Following Ref. [2] the $F$, il energy $E_{\mathrm{F}}$ for electrons of a given effective mass $\mu$ is

$$
E_{\mathrm{F}}=\left(\frac{m_{0}}{\mu}\right) \frac{\hbar^{2}}{2 m_{0}}\left(3 \pi^{2} n_{0}\right)^{2 / 3} .
$$

After converting Eqs. (1) and (2), and inserting to (5) we obtain a relation which allows to calculate $E_{\mathrm{F}}$ for a given energy loss $\Delta E$

$$
E_{\mathrm{F}}=B_{0}\left(\frac{m_{0}}{\mu}\right)^{1 / 3}(\Delta E)^{4 / 3}
$$

where $B_{0}=\frac{1}{2}\left(\frac{3}{4} \pi \hbar\right)^{2 / 3} e^{-4 / 3} m_{0}^{-1 / 3}=0.295 \mathrm{eV}^{-1 / 3}$.

A width of an energy band is given by

$$
\delta E=\frac{2 \hbar^{2}}{\mu a^{2}}
$$

where $a$ is the interatomic distance.

On the basis of Ref. [2] and Eq. (6) upon some converting we obtain a bandwidth expressed by an energy loss $\Delta E$

$$
\delta E=B_{0}\left(\frac{m_{0}}{\mu} \Delta E\right)^{4 / 3} .
$$

Conductivity of a solid state can be expressed as

$$
\gamma=\frac{n_{0} e^{2} \bar{\lambda}}{2 m_{0} \bar{u}}
$$

where $\bar{\lambda}$ is the mean free path length and $\bar{u}$ is the mean velocity.

Upon converting the obtained Eqs. (1), (3) and inserting into Eq. (9), and in view of a fact that in a classical model $\langle\bar{u} / \bar{\lambda}\rangle=\bar{d} \approx 3 \times 10^{13} \mathrm{~s}^{-1}$ we obtain

$$
\gamma=c(\Delta E)^{2}
$$

where $c=1 / \hbar^{2} 8 \pi \bar{d}=3.06 \times 10^{15} \mathrm{eV}^{-2} \mathrm{~s}^{-1}$. This relation allows to evaluate conductivity $\gamma$ knowing the energy loss $\Delta E$.

\section{Results}

The aim of the present work was to calculate the effective mass $\mu$, the bandwidth $\delta E$, the Fermi energy $E_{\mathrm{F}}$ and conductivity $\gamma$ for $3 d$ - and $4 d$-transition metals, crystalline alloys of the metals and the amorphous alloy $\mathrm{Fe}_{78} \mathrm{Si}_{9} \mathrm{~B}_{13}$. Values of characteristic energy losses $\Delta E$ were published in Refs. [3-9]. The calculations were made using relations (4), (6), (8) and (10) and the obtained results are given in Tabs. I-IV. Some values for the calculated quantities taken from literature [10-17] are also given for comparison. 


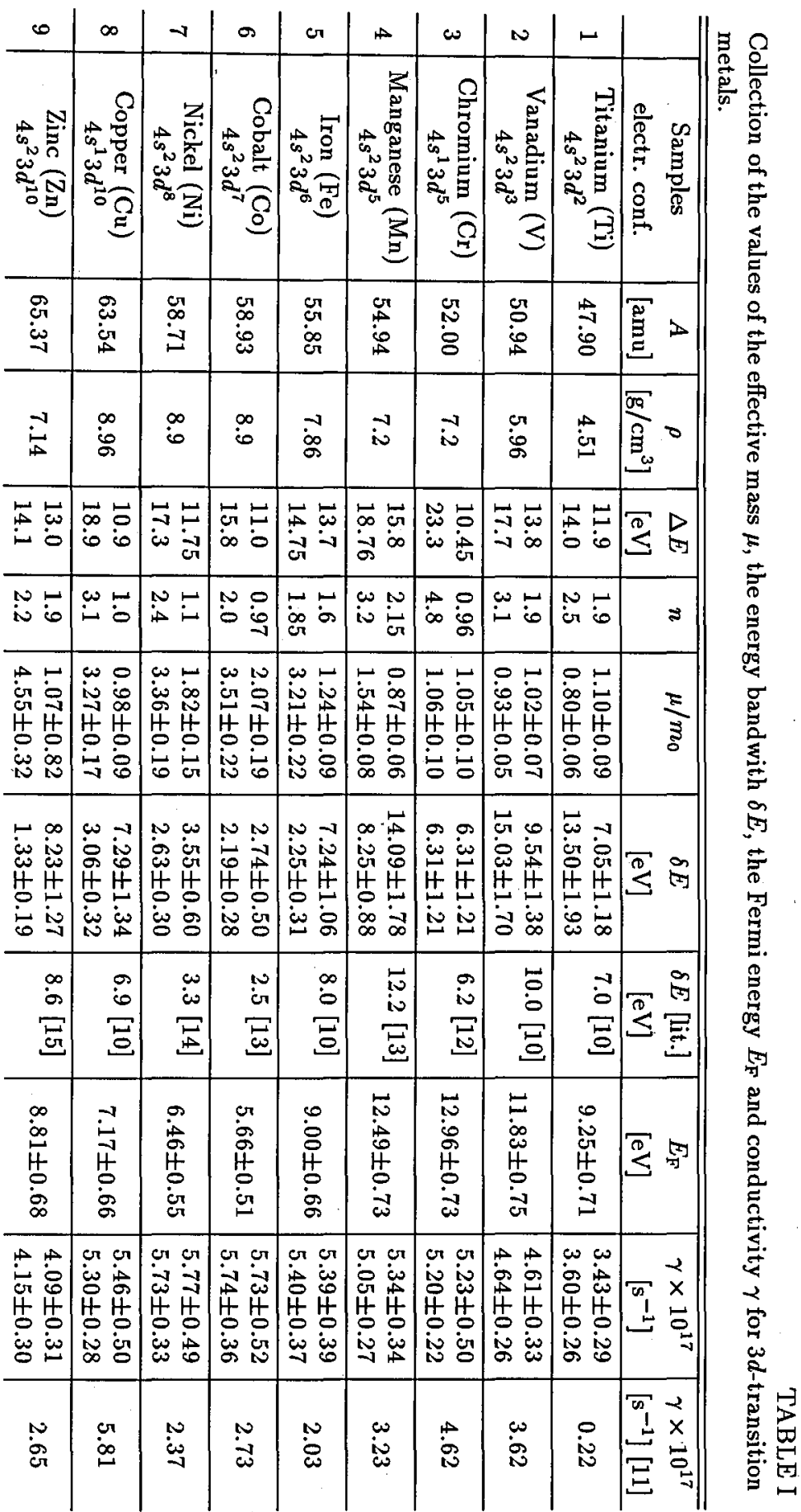




\begin{tabular}{|c|c|c|c|c|c|c|c|c|c|c|}
\hline$\omega$ & $\infty$ & $\rightarrow$ & 0 & G & $\Delta$ & 山 & $\omega$ & 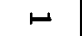 & & \\
\hline 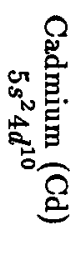 & 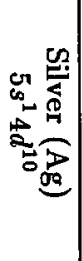 & 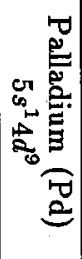 & 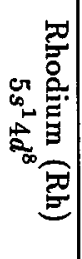 & 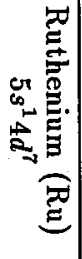 & 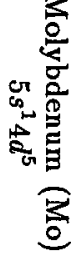 & 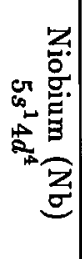 & 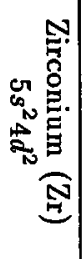 & 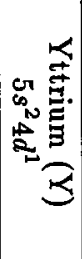 & 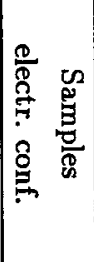 & 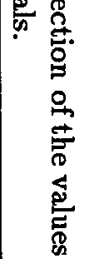 \\
\hline$\stackrel{\text { 卢 }}{\text { 点 }}$ & $\begin{array}{l}\text { 句 } \\
0 \\
0\end{array}$ & $\begin{array}{l}\overrightarrow{8} \\
\stackrel{8}{\circ}\end{array}$ & 总 & $\stackrel{5}{0}$ & 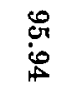 & $\begin{array}{l}0 \\
0 \\
0\end{array}$ & $\stackrel{\mathscr{N}}{\text { N }}$ & $\begin{array}{l}\infty \\
\infty \\
0 \\
0 \\
10\end{array}$ & D & $\begin{array}{l}\vec{\sigma} \\
0 \\
0\end{array}$ \\
\hline $\begin{array}{l}\infty \\
\dot{\phi}\end{array}$ & $\overbrace{i r}$ & $\underset{\sigma}{\sigma}$ & $\stackrel{\mathfrak{n}}{i}$ & 客 & 它 & 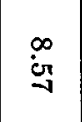 & i & cr & $\overbrace{\omega}^{\sigma 0}$ & 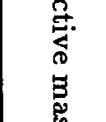 \\
\hline $\begin{array}{l}\vec{\sigma} \\
\dot{\sigma}\end{array}$ & $\vec{\bullet} \bullet$ & $\begin{array}{ll}\omega & \infty \\
i & i\end{array}$ & $\begin{array}{ll}\vec{v} & 0 \\
i & 0\end{array}$ & $\begin{array}{ll}\text { 供 } \\
\text { is } \infty\end{array}$ & $\stackrel{\infty}{\infty}_{\infty}^{0}$ & $\begin{array}{ll}\vec{v} & \infty \\
\dot{\sigma} & \infty\end{array}$ & 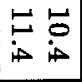 & 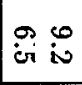 & $\stackrel{D}{\leq}$ & 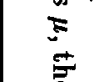 \\
\hline $\begin{array}{l}\omega \\
\infty \\
\infty\end{array}$ & 芯 & $\begin{array}{ll}N & 0 \\
\dot{\omega} & 0 \\
\dot{\omega} & 0 \\
0\end{array}$ & 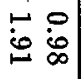 & $\begin{array}{ll}1 & 0 \\
\dot{0} & 0 \\
\dot{G} & \infty\end{array}$ & 08 & 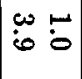 & $\stackrel{\sim}{\sim} \stackrel{\infty}{\infty}$ & $\stackrel{0}{\circ}$ & 2 & \\
\hline 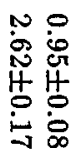 & 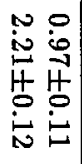 & 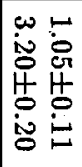 & 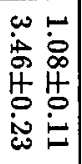 & 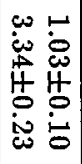 & 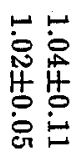 & $\begin{array}{ll}1 & 0 \\
0 & 0 \\
0 & 0 \\
1 & 0 \\
0 & 0 \\
\dot{*} & 0 \\
1 & .\end{array}$ & 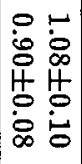 & 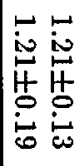 & & \\
\hline 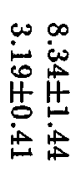 & 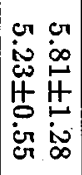 & 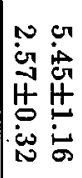 & 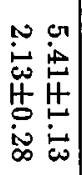 & 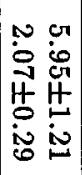 & 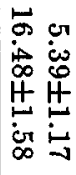 & 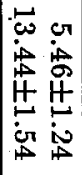 & 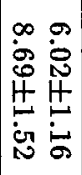 & 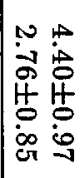 & $\stackrel{\infty}{\leq} \infty$ & 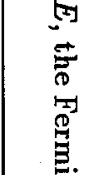 \\
\hline$\stackrel{\infty}{\vec{\sigma}}$ & 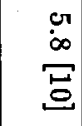 & 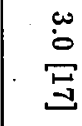 & 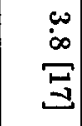 & 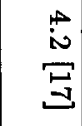 & $\stackrel{+}{\infty}$ & $\begin{array}{l}0 \\
\stackrel{0}{\sigma} \\
\stackrel{0}{0}\end{array}$ & $\begin{array}{l}\sigma \\
\ddot{\sigma} \\
\ddot{\Xi}\end{array}$ & $\begin{array}{l}\stackrel{N}{\sim} \\
\stackrel{\vec{\sigma}}{\sigma}\end{array}$ & 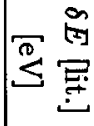 & 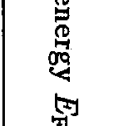 \\
\hline $\begin{array}{l}-1 \\
\infty \\
0 \\
0 \\
0 \\
0 \\
\infty\end{array}$ & $\begin{array}{l}\text { cr } \\
\dot{g} \\
\stackrel{H}{+1} \\
0 \\
\dot{S}\end{array}$ & 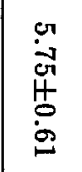 & $\begin{array}{l}0 \\
0 \\
0 \\
\stackrel{H}{+} \\
0 \\
\dot{0}\end{array}$ & $\begin{array}{l}\stackrel{\rho}{\omega} \\
\stackrel{\omega}{\oplus} \\
\stackrel{+}{\dot{\omega}}\end{array}$ & 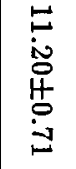 & $\begin{array}{l}\dot{0} \\
\text { i } \\
0 \\
1 \\
0 \\
8\end{array}$ & $\begin{array}{l}\overrightarrow{-1} \\
\dot{\infty} \\
\stackrel{+}{0} \\
\dot{8}\end{array}$ & 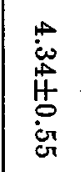 & $\sum^{\infty}$ & . \\
\hline 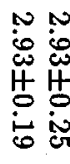 & 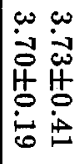 & 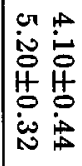 & 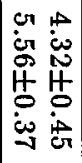 & 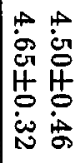 & 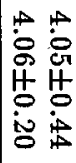 & 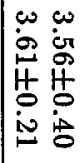 & 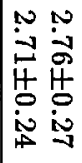 & 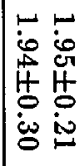 & 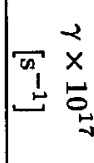 & 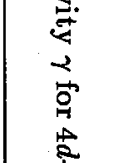 \\
\hline 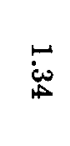 & :0 & 畓 & $\stackrel{\omega}{\omega}$ & $\stackrel{N}{\infty}$ & : & $\stackrel{N}{\circ}$ & 苫 & نั & 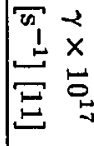 & 胥 \\
\hline
\end{tabular}


TABLE III

Collection of the values of the effective mass $\mu$, the energy bandwith $\delta E$, the Fermi energy $E_{\mathrm{F}}$ and conductivity $\gamma$ for metal alloys.

\begin{tabular}{|c|c|c|c|c|c|c|c|c|c|}
\hline & Samples & $\begin{array}{c}A \\
{[\mathrm{amu}]}\end{array}$ & $\stackrel{\rho}{\left[\mathrm{g} / \mathrm{cm}^{3}\right]}$ & $\begin{array}{c}\Delta E \\
{[\mathrm{eV}]}\end{array}$ & $n$ & $\mu / m_{0}$ & $\begin{array}{c}\delta E \\
{[\mathrm{eV}]}\end{array}$ & $\begin{array}{c}E_{\mathrm{F}} \\
{[\mathrm{eV}]}\end{array}$ & $\begin{array}{c}\gamma \times 10^{17} \\
{\left[\mathrm{~s}^{-1}\right]}\end{array}$ \\
\hline 1 & $F e_{1} V_{99}$ & 50.99 & 5.98 & $\begin{array}{l}11.3 \\
12.3\end{array}$ & $\begin{array}{l}1.31 \\
1.56 \\
\end{array}$ & $\begin{array}{l}1.52 \pm 0.13 \\
1.95 \pm 0.16\end{array}$ & $\begin{array}{l}4.27 \pm 0.76 \\
3.45 \pm 0.56\end{array}$ & $6.61 \pm 0.57$ & $\begin{array}{l}4.48 \pm 0.40 \\
4.46 \pm 0.36\end{array}$ \\
\hline 2 & $\mathrm{Fe}_{15} \mathrm{~V}_{85}$ & 51.68 & 6.25 & $\begin{array}{l}10.3 \\
13.8 \\
\end{array}$ & $\begin{array}{l}1.06 \\
1.90 \\
\end{array}$ & $\begin{array}{l}1.89 \pm 0.18 \\
1.81 \pm 0.13\end{array}$ & $\begin{array}{l}2.84 \pm 0.55 \\
4.42 \pm 0.64 \\
\end{array}$ & $6.68 \pm 0.55$ & $\begin{array}{l}4.60 \pm 0.45 \\
4.61 \pm 0.33\end{array}$ \\
\hline 3 & $\mathrm{Fe}_{32.21} \mathrm{~V}_{67.79}$ & 52.52 & 6.57 & $\begin{array}{r}9.8 \\
12.6 \\
\end{array}$ & $\begin{array}{l}0.93 \\
1.53\end{array}$ & $\begin{array}{l}2.16 \pm 0.22 \\
2.59 \pm 0.21\end{array}$ & $\begin{array}{l}2.22 \pm 0.45 \\
2.43 \pm 0.39\end{array}$ & $5.55 \pm 0.50$ & $\begin{array}{l}4.75 \pm 0.48 \\
4.77 \pm 0.38\end{array}$ \\
\hline 4 & $\mathrm{Fe}_{50} \mathrm{~V}_{50}$ & 53.39 & 6.91 & $\begin{array}{r}8.9 \\
13.2 \\
\end{array}$ & $\begin{array}{l}0.74 \\
1.63 \\
\end{array}$ & $\begin{array}{l}2.71 \pm 0.30 \\
2.77 \pm 0.21\end{array}$ & $\begin{array}{l}1.44 \pm 0.32 \\
2.37 \pm 0.36\end{array}$ & $5.23 \pm 0.47$ & $\begin{array}{l}4.92 \pm 0.55 \\
4.91 \pm 0.37\end{array}$ \\
\hline 5 & $\mathrm{Fe}_{66.7} \mathrm{~V}_{33.3}$ & 54.21 & 7.23 & $\begin{array}{l}11.0 \\
14.8\end{array}$ & $\begin{array}{l}1.10 \\
1.98\end{array}$ & $\begin{array}{l}1.83 \pm 0.17 \\
2.52 \pm 0.17\end{array}$ & $\begin{array}{l}3.23 \pm 0.59 \\
3.12 \pm 0.42\end{array}$ & $6.89 \pm 0.54$ & $\begin{array}{l}5.06 \pm 0.46 \\
5.08 \pm 0.34\end{array}$ \\
\hline 6 & $\mathrm{Fe}_{85} \mathrm{~V}_{15}$ & 55.11 & 7.58 & $\begin{array}{r}9.6 \\
12.2 \\
\end{array}$ & $\begin{array}{l}0.81 \\
1.31\end{array}$ & $\begin{array}{l}2.47 \pm 0.26 \\
4.25 \pm 0.35\end{array}$ & $\begin{array}{l}1.80 \pm 0.38 \\
1.21 \pm 0.20\end{array}$ & $4.79 \pm 0.44$ & $\begin{array}{l}5.23 \pm 0.55 \\
5.22 \pm 0.43\end{array}$ \\
\hline 7 & $\mathrm{Zn}_{10} \mathrm{Ni} 90$ & 59.38 & 8.72 & $\begin{array}{l}11.75 \\
17.0\end{array}$ & $\begin{array}{l}1.14 \\
2.38\end{array}$ & $\begin{array}{l}1.76 \pm 0.15 \\
3.45 \pm 0.20\end{array}$ & $\begin{array}{l}3.70 \pm 0.63 \\
2.47 \pm 0.30\end{array}$ & $6.52 \pm 0.56$ & $\begin{array}{l}5.57 \pm 0.47 \\
5.58 \pm 0.33\end{array}$ \\
\hline 8 & $\mathrm{Zn}_{20} \mathrm{Ni} 80$ & 60.04 & 8.55 & $\begin{array}{l}12.0 \\
16.7\end{array}$ & $\begin{array}{l}1.22 \\
2.36\end{array}$ & $\begin{array}{l}1.64 \pm 0.14 \\
3.55 \pm 0.21\end{array}$ & $\begin{array}{l}4.20 \pm 0.70 \\
2.32 \pm 0.28\end{array}$ & $6.87 \pm 0.57$ & $\begin{array}{l}5.42 \pm 0.45 \\
5.43 \pm 0.33\end{array}$ \\
\hline 9 & $\mathrm{Zn}_{27} \mathrm{Ni}_{73}$ & 60.51 & 8.42 & $\begin{array}{l}12.25 \\
16.5\end{array}$ & $\begin{array}{l}1.30 \\
2.36\end{array}$ & $\begin{array}{l}1.54 \pm 0.13 \\
3.62 \pm 0.22\end{array}$ & $\begin{array}{l}4.70 \pm 0.77 \\
2.23 \pm 0.27\end{array}$ & $7.22 \pm 0.59$ & $\begin{array}{l}5.31 \pm 0.43 \\
5.30 \pm 0.32\end{array}$ \\
\hline 10 & $\mathrm{Zn}_{32} \mathrm{Ni}_{68}$ & 60.84 & 8.34 & $\begin{array}{l}11.0 \\
15.0\end{array}$ & $\begin{array}{l}1.07 \\
1.98\end{array}$ & $\begin{array}{l}1.88 \pm 0.17 \\
4.36 \pm 0.29\end{array}$ & $\begin{array}{l}3.12 \pm 0.57 \\
1.53 \pm 0.20\end{array}$ & $5.85 \pm 0.53$ & $\begin{array}{l}5.20 \pm 0.47 \\
5.22 \pm 0.35\end{array}$ \\
\hline
\end{tabular}

TABLE IV

Collection of the values of the effective mass $\mu$, the energy bandwith $\delta E$, the Fermi energy $E_{\mathrm{F}}$ and conductivity $\gamma$ for the amorphous alloy $\mathrm{Fe}_{78} \mathrm{Si}_{9} \mathrm{~B}_{13}$ annealed for $4 \mathrm{~h}$ as a function of temperature.

\begin{tabular}{|c|c|c|c|c|c|c|c|c|c|}
\hline & $\begin{array}{l}\text { Anneal. } \\
\text { temp. [K] }\end{array}$ & $\begin{array}{c}\boldsymbol{A} \\
{[\mathrm{amu}]}\end{array}$ & $\stackrel{\rho}{\left[\mathrm{g} / \mathrm{cm}^{3}\right]}$ & $\begin{array}{c}\Delta E \\
{[\mathrm{eV}]}\end{array}$ & $n$ & $\mu / m_{0}$ & $\begin{array}{c}\delta E \\
{[\mathrm{eV}]}\end{array}$ & $\begin{array}{c}E_{\mathrm{F}} \\
{[\mathrm{eV}]}\end{array}$ & $\begin{array}{c}\gamma \times 10^{17} \\
{\left[\mathrm{~s}^{-1}\right]}\end{array}$ \\
\hline 1 & 573 & \multirow{8}{*}{47.49} & \multirow{8}{*}{6.65} & 7.8 & 0.51 & $3.81 \pm 0.49$ & $0.77 \pm 0.20$ & \multirow{2}{*}{$2.92 \pm 0.37$} & $5.48 \pm 0.70$ \\
\hline 1 & 573 & & & 10.4 & 0.82 & $5.02 \pm 0.48$ & $0.78 \pm 0.15$ & & $6.06 \pm 0.58$ \\
\hline \multirow{2}{*}{2} & \multirow{2}{*}{673} & & & 8.0 & 0.52 & $3.62 \pm 0.45$ & $0.85 \pm 0.21$ & \multirow{2}{*}{$3.07 \pm 0.38$} & $5.66 \pm 0.71$ \\
\hline & & & & 10.8 & 0.85 & $4.65 \pm 0.43$ & $0.91 \pm 0.17$ & & $6.31 \pm 0.58$ \\
\hline \multirow{2}{*}{3} & \multirow{2}{*}{723} & & & 7.6 & 0.50 & $4.02 \pm 0.53$ & $0.69 \pm 0.18$ & \multirow{2}{*}{$2.77 \pm 0.36$} & $5.31 \pm 0.70$ \\
\hline & & & & 10.2 & 0.80 & $5.22 \pm 0.51$ & $0.72 \pm 0.14$ & & $5.98 \pm 0.59$ \\
\hline \multirow{2}{*}{4} & \multirow{2}{*}{773} & & & 7.2 & 0.47 & $4.47 \pm 0.62$ & $0.56 \pm 0.15$ & \multirow{2}{*}{$2.49 \pm 0.35$} & $5.07 \pm 0.70$ \\
\hline & & & & 9.4 & 0.73 & $6.14 \pm 0.65$ & $0.52 \pm 0.11$ & & $5.56 \pm 0.59$ \\
\hline
\end{tabular}

\section{Conclusions}

Among transition metals we may distinguish a group of metals where both $s$ and $d$ electrons behave as free electrons. $\mathrm{Ti}, \mathrm{V}, \mathrm{Cr}, \mathrm{Zr}, \mathrm{Nb}$, and Mo belong to this group. In the rest metals $d$ electrons are more strongly bonded than $s$ electrons. In the latter group we may distinguish $\mathrm{Fe}, \mathrm{Co}$, and $\mathrm{Ni}$. The effective mass $\mu$ in them is remarkably different from the electron rest mass.

Also in alloys a mean free path length of $s$ electrons is restricted and $d$ electrons are more strongly bonded than $s$ electrons. Particularly in the amorphous 
alloy $\mathrm{Fe}_{78} \mathrm{Si}_{9} \mathrm{~B}_{13}$ the interaction of $s$ and $d$ electrons with atomic cores is quite strong although they are not as strongly bonded as core electrons.

From the above consideration it is concluded that $d$ electrons in all investigated samples, despite their restricted freedom, still do not belong to an atomic core. They may participate in conduction.

The values of conductivity for transition metals and their alloys suggest that contribution of $s$ and $d$ electrons to conduction is comparable.

Thus metals with poorly filled $d$ band and those with $d$ band completely filled are characterized by smaller conductivity.

In majority of cases the obtained results for the bandwidth $\delta E$ and conductivity $\gamma$ are in good agreement with literature data. This allowed us to apply similar calculations of the above quantities for alloys of those metals in polycrystalline and amorphous phase (Tables III and IV).

\section{References}

[1] D. Pines, Usp. Fiz. Nauk 62, 399 (1957).

[2] W.A. Harrison, Solid State Theory, Mc Graw-Hill, New York 1970.

[3] H.B. Kolodziej, J. Wesołowski, B. Rozenfeld, Acta Phys. Pol. A 47, 751 (1975).

[4] H.B. Kolodziej, B. Rozenfeld, Acta Phys. Pol. A 48, 765 (1975).

[5] H.B. Kolodziej, J. Olesik, U. Madałkiewicz, Acta Phys. Pol. A 59, 443 (1981).

[6] H.B. Kolodziej, Acta Phys. Pol. A 66, 261 (1984).

[7] H.B. Kolodziej, Acta Phys. Pol. A 64, 617 (1983).

[8] H.B. Kołodziej, B. Rozenfeld, A. Kolarz, R. Szczęsny, Acta Phys. Pol. A 50, 21 (1976).

[9] H.B. Kołodziej, E. Jakubczyk, Z. Mandecki, B. Całusiriski, Acta Phys. Pol. A 83, 327 (1993).

[10] S. Toudaard, J. Kraaer, Phys. Rev. B 43, 1651 (1991).

[11] F.J. Blatt, Physics of Electronic Conduction in Solids, McGraw-Hill, New York 1972, p. 217.

[12] E. Bertel, Surf. Sci. 152/153, 776 (1985).

[13] J. Fink, Th. Müller-Heinzerling, B. Scheerer, Phys. Rev. B 32, 4899 (1985).

[14] T. Okuri, H. Ishioka, H. Kukuda, M. Irako, J. Phys. Soc. Jap. 55, 414 (1986).

[15] V.D. Gorobchenko, M.V. Zharnikov, E.G. Maksimov, S.N. Rashkeyev, Zh. Eksp. Teor. Fiz. 88, 677 (1985).

[16] Y. Baba, T.A. Sasaki, Surf. Interface Anal. 6, 171 (1984).

[17] I.I. Mazin, E.G. Maksimov, S.N. Rashkeyev, Yu.A. Uspensky, Zh. Eksp. Teor. Fiz. 90, 1092 (1986). 